\section{Two distinct modes for propagation of histone PTMs across the cell cycle}

\author{
Constance Alabert, ${ }^{1,2,7}$ Teresa K. Barth, ${ }^{3,4,7}$ \\ Nazaret Reverón-Gómez, ${ }^{1,2}$ Simone Sidoli, ${ }^{2,5,6}$ \\ Andreas Schmidt, ${ }^{3,4}$ Ole N. Jensen, ${ }^{2,5}$ Axel Imhof, ${ }^{3,4}$ \\ and Anja Groth ${ }^{1,2}$
}

\begin{abstract}
${ }^{1}$ Biotech Research and Innovation Centre (BRIC), ${ }^{2}$ Centre for Epigenetics, University of Copenhagen, 2200 Copenhagen, Denmark; ${ }^{3}$ Munich Centre of Integrated Protein Science, ${ }^{4}$ Adolf Butenandt Institute, Ludwig-Maximillians University of Munich, 80336 Munich, Germany; ${ }^{5}$ Department of Biochemistry and Molecular Biology, University of Southern Denmark, DK-5230 Odense, Denmark
\end{abstract}

Epigenetic states defined by chromatin can be maintained through mitotic cell division. However, it remains unknown how histone-based information is transmitted. Here we combine nascent chromatin capture (NCC) and triple-SILAC (stable isotope labeling with amino acids in cell culture) labeling to track histone modifications and histone variants during DNA replication and across the cell cycle. We show that post-translational modifications (PTMs) are transmitted with parental histones to newly replicated DNA. Di- and trimethylation marks are diluted twofold upon DNA replication, as a consequence of new histone deposition. Importantly, within one cell cycle, all PTMs are restored. In general, new histones are modified to mirror the parental histones. However, H3K9 trimethylation (H3K9me3) and $\mathrm{H} 3 \mathrm{~K} 27 \mathrm{me} 3$ are propagated by continuous modification of parental and new histones because the establishment of these marks extends over several cell generations. Together, our results reveal how histone marks propagate and demonstrate that chromatin states oscillate within the cell cycle.

Supplemental material is available for this article.

Received November 28, 2014; revised version accepted February 18, 2015.

Local chromatin environment is a major determinant of gene expression and underlies epigenetic phenomena such as $\mathrm{X}$ inactivation, gene silencing, and centromere function. Chromatin states can thus be maintained through cell division but, at the same time, must be sufficiently plastic to allow programmed changes in transcription patterns during development. Aberrant epigenetic regulation is prevalent in complex diseases, and cancer

[Keywords: histone post-translational modifications; DNA replication; cell cycle; histone variants; epigenetics; histone recycling]

${ }^{6}$ Present address: Epigenetics Program, Department of Biochemistry and Biophysics, Perelman School of Medicine, University of Pennsylvania, Philadelphia, PA 19104, USA.

${ }^{7}$ These authors contributed equally to this work.

Corresponding authors: imhof@lmu.de, anja.groth@bric.ku.dk

Article is online at http://www.genesdev.org/cgi/doi/10.1101/gad.256354. 114. cells typically show widespread chromatin alterations. Thus, defining the mechanisms underpinning the propagation of chromatin states is important for understanding cellular memory and disease.

Histones are highly decorated with post-translational modifications (PTMs) that can directly affect chromatin accessibility or serve as a recognition site for specific proteins. Some histone marks are closely linked to gene activation, and others are required to maintain silencing (Kouzarides 2007; Pengelly et al. 2013). In addition, histone variants occupying distinct genomic regions also define chromatin structure (Skene and Henikoff 2013). For example, histones H3.3 and H2A.Z generally demarcate dynamic sites with high nucleosome turnover. During DNA replication, nucleosomes are disrupted ahead of the replication machinery and reassembled on the two newly synthesized DNA strands by the recycling of old histones and incorporation of new largely unmodified histones (Annunziato 2005; Probst et al. 2009; Margueron and Reinberg 2010; Alabert and Groth 2012). The recycling of modified parental histones offers an attractive epigenetic mechanism, as it ensures that histone marks are kept at their correct location on newly replicated DNA. Moreover, parental histones may also serve as a blueprint to modify neighboring new histones, given that modifications like $\mathrm{H} 3 \mathrm{~K} 9$ trimethylation (H3K9me3) and $\mathrm{H} 3 \mathrm{~K} 27 \mathrm{me} 3$ can recruit their cognate enzyme and potentially be self-propagating (Aagaard et al. 1999; Hansen et al. 2008; Margueron et al. 2009). However, paradoxically, for several key modifications, mass spectrometry analysis showed that new histones had not acquired modifications to become identical to the old parental histones in the G1 phase of the next cell cycle (Scharf et al. 2009; Sweet et al. 2010; Xu et al. 2012). Besides, recent work in Drosophila embryos challenges the paradigm that histone marks are transmitted with the parental histones by suggesting that the marks are erased (Petruk et al. 2012, 2013). Thus, it remains unclear how histone PTM levels are restored after DNA replication to propagate epigenetic states through multiple rounds of cell divisions.

\section{Results and Discussion}

Histones are recycled with their PTMs at replication forks

We tracked newly synthesized and parental histones by quantitative mass spectrometry immediately after their deposition behind the replication fork using nascent chromatin capture (NCC). In NCC, biotin-dUTP labeling of newly replicated DNA and cross-linking of associated proteins allow the isolation of nascent chromatin (Supplemental Fig. S1A,B; Alabert et al. 2014). We combined NCC with pulsed SILAC (stable isotope labeling with amino acids in cell culture) to differentiate newly synthesized histones (heavy) and old recycled histones (light) (Fig. 1A; Scharf et al. 2009). The nascent chromatin purified by NCC contained approximately

(C) 2015 Alabert et al. This article is distributed exclusively by Cold Spring Harbor Laboratory Press for the first six months after the full-issue publication date (see http://genesdev.cshlp.org/site/misc/terms.xhtml). After six months, it is available under a Creative Commons License (Attribution-NonCommercial 4.0 International), as described at http:// creativecommons.org/licenses/by-nc/4.0/. 
A

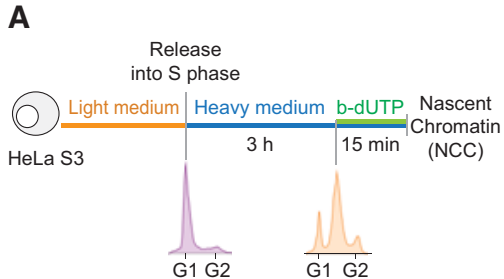

B

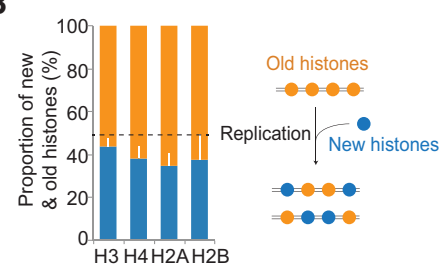

C

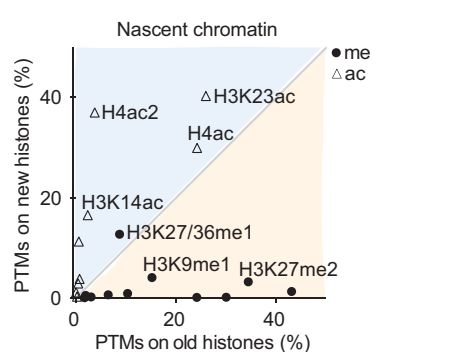

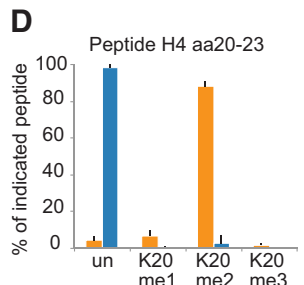

E

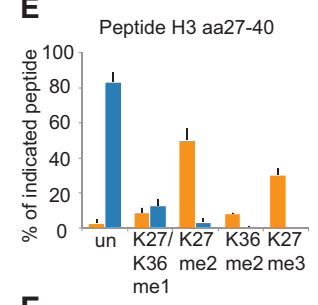

$\mathbf{F}$

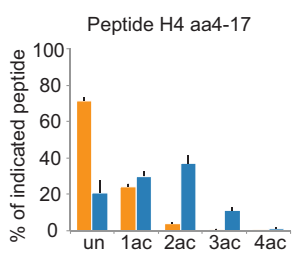

Figure 1. Histones are recycled together with their modifications on newly replicated DNA. (A) Experimental design. Cells were synchronized by thymidine and released in heavy SILAC medium. Newly replicated DNA was labeled with b-dUTP and isolated on streptavidin beads by NCC (Supplemental Fig. S1A). (B) Proportion of new and old histones on nascent chromatin. The dotted line indicates theoretical values. (C) Overview of how histone PTMs in nascent chromatin distribute on new and old histones. The mean of nine independent biological experiments is shown $(n=9)$. Old histones are mainly methylated (me), while new ones are acetylated (ac). Only shared marks are labeled. For values for all identified PTMs, see Supplemental Figure S1C. $(D-F)$ Modifications on new (blue) and old (orange) histone $\mathrm{H} 3$ and $\mathrm{H} 4$ in nascent chromatin shown as a percentage of heavy or light peptides, respectively. The $\mathrm{H} 4$ amino acid 4-17 (aa4-17) peptide contains K5, K8, K12, and K16. (Un) Unmodified; (ac) acetylated; (ac2) diacetylated; (ac3) triacetylated; (ac4) quadriacetylated; (me1) monomethylated; (me2) dimethylated; (me3) trimethylated. K27/K36me1 is resolved in Supplemental Figure S1, E and F.

equal amounts of new and old histones (Fig. 1B). The slight overrepresentation of old histones likely reflects a contribution of recycled light amino acids during histone biosynthesis (Scharf et al. 2009; Xu et al. 2012; Zee et al. 2012). Thus, chromatin is reassembled by a combination of recycled parental histones and newly synthesized ones.

New and old histones remained markedly different within the first minutes following their assembly on newly replicated DNA (Fig. 1C; Supplemental Fig. S1C). Old recycled histones presented a large variety of PTMs, including mono-, di-, and trimethylation (Fig. 1D,E). Importantly, no marks were underrepresented on old histones in nascent chromatin compared with the whole genome (Supplemental Fig. S1D), arguing that histone marks are efficiently transmitted during DNA replication. Thus, in human somatic cells, there is no global replication-coupled erasure of parental histone PTMs, as proposed for Drosophila embryos (Petruk et al. 2012, 2013). New histone $\mathrm{H} 4$ showed high levels of diacetylation (Fig. 1F; Supplemental Fig. S1C), a hallmark of newly synthesized histone H4 (Sobel et al. 1995; Loyola et al. 2006; Jasenca-

kova et al. 2010). New histone H3 carried H3K14ac, $\mathrm{H} 3 \mathrm{~K} 18 / \mathrm{K} 23 \mathrm{ac}$, and $\mathrm{H} 3 \mathrm{~K} 9 \mathrm{me} 1$ at levels closely mirroring the modification pattern of soluble histone $\mathrm{H} 3$ in predeposition complexes (Supplemental Fig. S1C; Loyola et al. 2006; Jasencakova et al. 2010), suggesting that these PTMs are maintained within the first minutes of incorporation. Notably, there is no enrichment of H3K56ac in the predeposition complex (Jasencakova et al. 2010) or in nascent chromatin (Supplemental Fig. S1C), arguing against a general function of this modification in chromatin assembly in human cells.

New histone H3 also carries modifications that were not present in predeposition complexes: K27me1 (8\%), K36me1 (4\%), and K27me2 (3\%) (Fig. 1E; Supplemental Fig. S1C). This suggests that these methylations are thus the first ones to be imposed on new histones after deposition onto newly replicated DNA. To confirm that H3K27me1 was imposed on new histones in nascent chromatin as also suggested previously (Benson et al. 2006), we blocked the production of new histones using cycloheximide treatment and measured H3K27mel levels by NCC (Supplemental Fig. S2A). We found that H3K27me1 levels were reduced upon cycloheximide treatment, consistent with rapid acquisition of H3K27mel on new histones (Supplemental Fig. S2B-E). We next performed similar experiments in early and late $\mathrm{S}$ phase in order to test whether our conclusions can be extended to all stages of S phase (Supplemental Fig. S3A). Indeed, since euchromatin is preferably replicated in early $\mathrm{S}$ phase and heterochromatin is preferably replicated in late $\mathrm{S}$ phase, it has been suggested that chromatin assembly may differ during S phase (Taddei et al. 1999; Zhang et al. 2002). We found that histone PTMs in nascent chromatin were largely
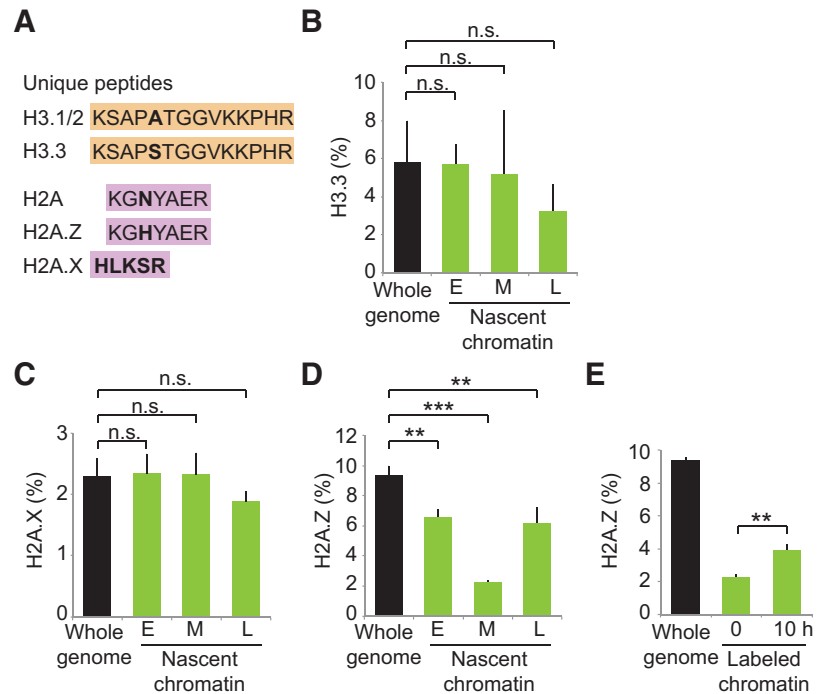

Figure 2. Recycling of histone variants at replication forks. Experimental design as in Figure 1A except that nascent chromatin was collected in early (E), mid- (M), and late (L) S phase (Supplemental Fig. S3A). (A) Unique peptides used to differentiate canonical histones $\mathrm{H} 3.1$ and $\mathrm{H} 3.2$ from the $\mathrm{H} 3.3$ variant as well as histone H2A from H2A.X and H2A.Z variants. (B) Enrichment of $\mathrm{H} 3.3$ relative to total $\mathrm{H} 3(\mathrm{H} 3.3+\mathrm{H} 3.1 / 2) .(C, D)$ Enrichment of H2A.X and H2A.Z relative to total H2A $(\mathrm{H} 2 \mathrm{~A}+\mathrm{H} 2 \mathrm{~A} \cdot \mathrm{Z}+\mathrm{H} 2 \mathrm{AX})$. (E) Enrichment of H2A.Z in labeled chromatin at $0 \mathrm{~h}$ (nascent; mid-S phase) and $10 \mathrm{~h}$ later (see Supplemental Fig. S4A for experimental design). Error bars indicate SD. $3<n<9$. Unpaired $t$-test: $\left.{ }^{* * *}\right) P<0.001$; $\left(^{* *}\right) P<0.05$; (n.s.) nonsignificant. 
similar in early and late S phase (Supplemental Fig. S3BD). However, K27me1/36me1 was increased, and H4 diacetylation was reduced on new histones incorporated in late S phase (Supplemental Fig. S3B). These observations, together with previous work (Taddei et al. 1999; Zhang et al. 2002; Lande-Diner et al. 2009), suggest a faster onset of silencing in late S phase.

\section{H2A.Z domains are challenged by the passage of replication forks}

In parallel, we explored the maintenance of histone variants during DNA replication. We used unique peptides to differentiate the canonical histone $\mathrm{H} 3.1$ from the variant $\mathrm{H} 3.3$ and the canonical histone $\mathrm{H} 2 \mathrm{~A}$ from the two variants H2A.X and H2A.Z (Fig. 2A). We found that H2A.X and H3.3 were equally as abundant in newly replicated chromatin as over the whole genome (Fig. 2B,C), arguing that they are not lost during replication. Indeed, analysis of old histones revealed that H2A.X and H3.3 are efficiently recycled at replication forks (Supplemental Fig. S3E). However, newly replicated regions were depleted for the histone variant H2A.Z (Fig. 2D). The low abundance of H2A.Z on newly replicated chromatin was not due to the particular composition of the replicated region, since we observed the same trend in early, mid, and late S phase (Fig. 2D). H2A.Z domains are thus challenged by the passage of replication forks. In-depth analysis of old and new histones showed that H2A.Z loss is the result of both inefficient recycling and lack of new deposition (Supplemental Fig. S3E,F). Notably, H2A.Z levels increased within the next $10 \mathrm{~h}$ (Fig. 2E), consistent with replication-independent assembly (Luk et al. 2010; Nekrasov et al. 2012). Altogether, our data demonstrate that histone variants are not recycled with similar efficiency at replication forks and that H2A.Z marked sites may not be maintained after DNA replication.

\section{Histone PTM levels are restored by two distinct modes}

Histone PTM levels must be maintained across the cell cycle (Probst et al. 2009; Margueron and Reinberg 2010; Alabert and Groth 2012; Zee et al. 2012). Previous analysis using SILAC labeling to differentiate new and old histones as well as methylation kinetics in total chromatin found that modification of new histones is a slow process extending into the next cell cycle (Pesavento et al. 2008; Scharf et al. 2009; Sweet et al. 2010; Xu et al. 2012; Zee et al. 2012). However, full restoration of the prereplication chromatin state could not be monitored, most likely due to limitations associated with analyzing total chromatin. Therefore, the mechanisms of restoration remain unclear. We thus investigated the kinetics of PTM establishment on new histones from the moment of their deposition and across several cell generations by combining NCC with double-pulsed (Supplemental Fig. S4A) and triplepulsed (Supplemental Fig. S5A) SILAC. The triple-pulsed SILAC allowed us to follow one generation of histones through several cell cycles (Supplemental Fig. S5B,C). Using the PTM level of the parental histones as an indicator of the prereplication state, we found that most methylations marks were diluted by twofold immediately after replication (Fig. 3A, 0 h; Supplemental Fig. S5D). However, within one cell cycle, the level of all PTMs was fully
A

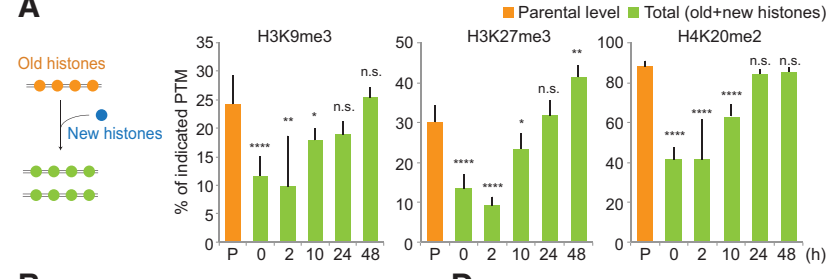

B

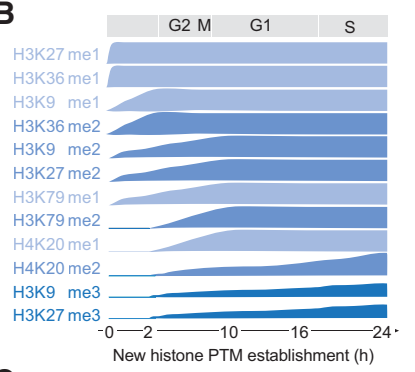

D
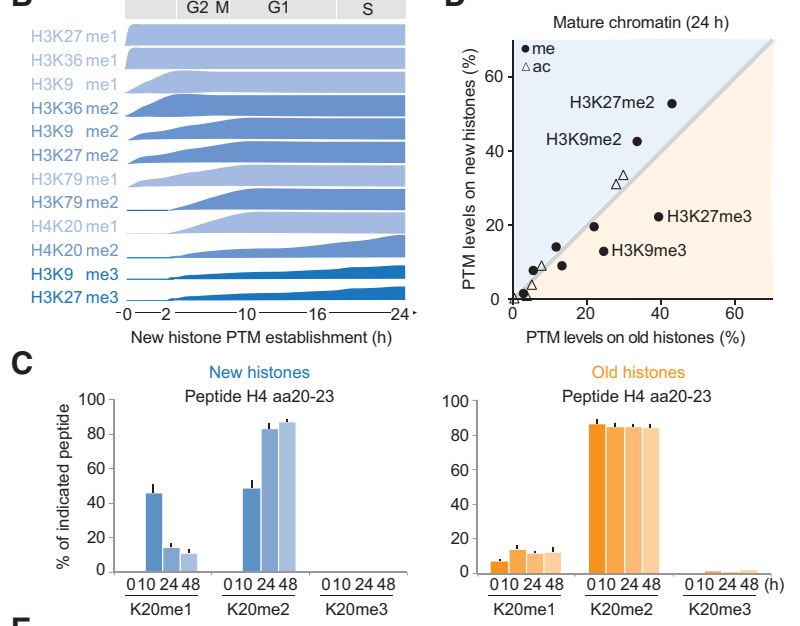

E
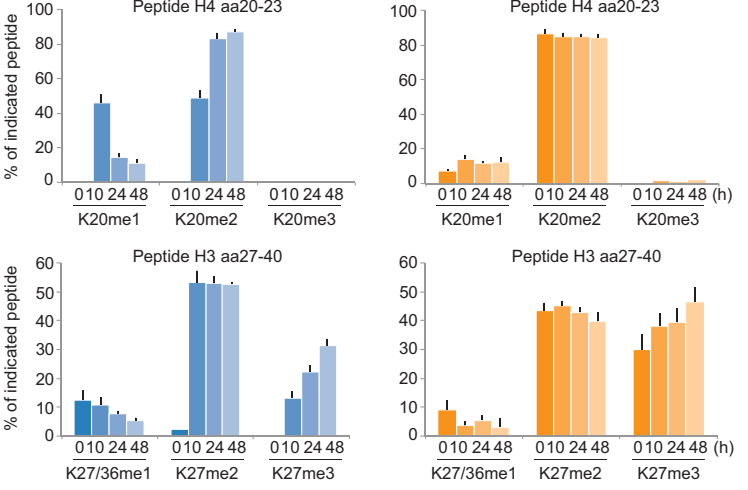

Figure 3. Propagation of histone PTMs across the cell cycle. Histone PTM propagation was determined by NCC coupled with double- and triple-SILAC labeling in a time-course analysis (Supplemental Figs. S4A, S5A). (A) Kinetics of PTM restoration. The total PTM level on replicated DNA (green) was compared with the PTM level of the old histones alone ( $\mathrm{P}$; orange), which, in nascent chromatin, represents the parental chromatin state that should to be reached by new and old histones together at the next round of replication, where they (new+old) constitute the parental histones of the daughter cells. After $24 \mathrm{~h}$, the total PTM level of new+old histones ( $24 \mathrm{~h}$; green) mirrors the parental histones of the previous round of replication $(\mathrm{P}$; orange), showing that the chromatin state is propagated. Error bars indicate SD. $n \geq 3$. Unpaired $t$-test identified differences from the parental state. A combination of double- and triple-SILAC time-course data is shown. $(B)$ Kinetics of PTM establishment on new histones. (C) Dynamics of H4K20 methylation on new and old histones. Error bars indicate SD. $n \geq 3$. (D) Overview of how histone PTMs in mature chromatin $(24 \mathrm{~h})$ distribute on new and old histones $(n=3)$. Old and new histones are identical except for the indicated marks. For values for all identified PTMs throughout the time course, $C$ and $E$, and Supplemental Figure S6, A and B. (E) Dynamics of H3K27 methylation on new and old histones. Error bars indicate SD. $n \geq 3$.

restored (Fig. 3A, 24 h; Supplemental Fig. S5D). Thus, in this system, we can address how PTM levels are maintained across the cell cycle.

We next examined the PTM levels on new and old histones during the maturation process. Since high histone turnover at gene bodies and regulatory elements was suggested to impact histone PTM maintenance (Deal et al. 2010), we first estimated the histone turnover in our system. We detected a turnover rate of $\sim 0.4 \%$ histone $\mathrm{H} 4$ per hour (Supplemental Fig. S5C), likely having a limited role in histone PTM maintenance for the replicated 
regions examined in our study. We found that for the majority of the histone PTMs, the prereplication level was restored by gradual modification of new histones until they became identical to the old histones (Fig. 3B; Supplemental Fig. S6A). Depending on the modification, the kinetics of PTM establishment varied from 2 to $24 \mathrm{~h}$ (Fig. 3B,C; Supplemental Figs. S4B-E, S6A), arguing against a general rapid replication-coupled restoration of histone PTMs. Notably, H3K9me3 and H3K27me3 remained underrepresented on new histones after $24 \mathrm{~h}$ (Fig. 3D), supporting previous findings (Xu et al. 2012) and indicating that restoration of these marks must occur by a different mechanism. Consistent with this, we found that both new and old histones acquired H3K27me3 and H3K9me3 (Fig. 3E; Supplemental Fig. S6B), together restoring the prereplication PTM level (Fig. 3A). This provides direct evidence for the model proposed by Zhu and colleagues (Xu et al. 2012; Huang et al. 2013) and argues against a simple copy-paste mechanism for propagation of histone PTMs. Strikingly, the addition of these trimethylation marks continues for up to three cell generations after histone incorporation (Fig. 3E; Supplemental Fig. S6B), and, consequently, H3K9me3 and H3K27me3 accumulate with histone age. Altogether, our findings define two distinct modes of PTMs maintenance: (1) For most PTMs (Fig. 3B), levels are maintained according to the simple paradigm that new histones acquire modifications to become identical to the old ones. (2) H3K9me3 and H3K27me3, marks central for cellular memory (Kouzarides 2007; Probst et al. 2009; Margueron and Reinberg 2010; Apostolou and Hochedlinger 2013), propagate by continuous modification of both new and old histones.

\section{Cell cycle withdrawal impacts histone PTM levels}

Collectively, our data argue that due to replication-coupled dilution of histone PTMs, chromatin states oscillate within the cell cycle. We thus investigated how active cycling impacts histone PTM levels. First, we blocked cells by nocodazole (Supplemental Fig. S7A) to determine whether PTM establishment on new histones requires passage through mitosis, as shown for incorporation of the histone variant CENP-A (Silva et al. 2012). We found that $\mathrm{H} 3 \mathrm{~K} 9 \mathrm{me} 3$ and $\mathrm{H} 3 \mathrm{~K} 27 \mathrm{me} 3$ acquisition was impeded in nocodazole (Fig. 4A; Supplemental Fig. S7B), suggesting that cell cycle regulation partly accounts for the slow establishment of these marks. Next, we tested whether exit from the cell cycle impacts histone methylation levels, as predicted from (1) the continuous PTM dilution in proliferating cells (Fig. 3A) and (2) the fact that older histones in general show a higher methylation state than new ones (Fig. 3E; Supplemental Fig. S6B). We used primary human TIG-3 fibroblasts to compare histone methylation levels in proliferating and G0-arrested cells (Fig. 4B; Supplemental Fig. S7C). H3K9 methylation did not accumulate further in G0-arrested cells, suggesting that accumulation of this modification requires an S-phase arrest as previously reported (Di Micco et al. 2011; Xu et al. 2012). However, for several other methylation marks, including $\mathrm{H} 3 \mathrm{~K} 27 \mathrm{me} 2 / 3$, H3K79me1/me2, and H4K20me2/me3, withdrawal from the cell cycle was indeed accompanied by a significant increase (Fig. 4B; Supplemental Fig. S7D). This suggests that histone PTM levels and possibly the stability of chromatin states are linked to cell cycle progression.

Our data demonstrate that histones PTMs are transmitted with high efficiency at replication forks. This explains the observation that $\mathrm{H} 3 \mathrm{~K} 27 \mathrm{me} 3$ can be transmitted through several cell generations in the absence of the enzyme (Gaydos et al. 2014). We show that chromatin states oscillate within the cell cycle due to replication-coupled dilution of histone PTMs. Whether the drop in histone PTM levels after DNA replication affects gene expression and could provide an opportunity to modify the transcription program remains an open question (Steffen and Ringrose 2014). We envision that it could provide a window of opportunity to rewire cell fate (Fisher and Mechali 2003; Tsubouchi et al. 2013). By following histones from the moment of their incorporation, we found that most PTMs are not imposed on new histones in a rapid replication-coupled manner. Instead, we identified two modes for PTM transmission across the cell cycle (Fig. 5). For H3K9me3 and H3K27me3, our data question the simple self-propagation mechanism in which PTMs on old histones are duplicated to neighboring new histones (Probst et al. 2009; Margueron and Reinberg 2010; Alabert and Groth 2012). The establishment of these trimethylation marks is slow and continues beyond several cell generations after histone deposition. However this does not lead to dilution or loss of $\mathrm{H} 3 \mathrm{~K} 27 \mathrm{me} 3$ and $\mathrm{H} 3 \mathrm{~K} 9 \mathrm{me} 3$, as the total levels are maintained across cell generations by the combined methylation of both new and old histones (Fig. 3A). The slow establishment of H3K9me3 and H3K27me3 on new histones could reflect that dimethylation-to-trimethylation conversion is not processive and 


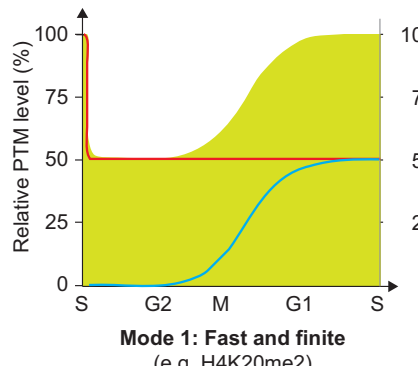

(e.g. H4K20me2)

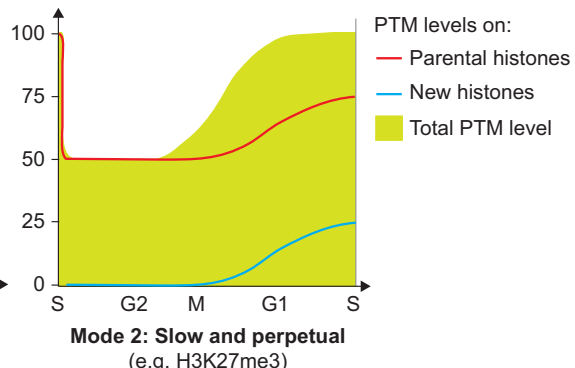

(e.g. H3K27me3)

Figure 5. Illustration of the two basic principles for histone PTM propagation. The contribution of new and parental histones (lines) to the total PTM level (green) is shown. (Mode 1) New histones acquire PTMs to become identical to the parental histones within one cell cycle (H3K9mel, H3K27/36me1, H3K36me2, H3K9me2, H3K27me2, H3K79me1, H3K79me2, H4K20me1, and $\mathrm{H} 4 \mathrm{~K} 20 \mathrm{me}$ ); (Mode 2) propagation relies on progressive modification of both new and parental histones (H3K9me3 and H3K27me3).

may require recruitment of enzyme cofactors (Alabert et al. 2014; Kalb et al. 2014). Such a tight regulation of PTM establishment could limit unwarranted silencing, which is jeopardized in cancers carrying EZH2-activating mutations (McCabe et al. 2012). Evidently, it will be important to consider the kinetics of PTM restoration in current models of epigenetic inheritance (Alabert and Groth 2012; Huang et al. 2013; Campos et al. 2014). We further envision that this mode of PTM maintenance could explain how cell cycle speed and withdrawal impact on cellular plasticity. DNA replication could be instrumental to reset epigenetic states (Tsubouchi et al. 2013), and, by revealing how histone PTMs are maintained across the cell cycle, our findings pave the way to understanding how cellular identity and reprogramming potential are connected with cell proliferation (Guo et al. 2014).

\section{Materials and methods}

\section{NCC}

Cells were synchronized by thymidine in light medium and released into $\mathrm{S}$ phase in heavy medium for $3 \mathrm{~h}$. Newly synthesized DNA was pulselabeled for 15 min with b-dUTP, and NCC was performed as described (Alabert et al. 2014). In brief, cells were labeled with b-dUTP for 5 min in hypotonic buffer (50 mM KCl, $10 \mathrm{mM} \mathrm{HEPES})$ and fixed 15 min later in $2 \%$ formaldehyde. Nuclei were isolated in sucrose buffer $(0.3 \mathrm{M}$ sucrose, $10 \mathrm{mM}$ HEPES-NaOH at $\mathrm{pH} 7.9,1 \%$ Triton X-100, 2 mM MgOAc), and chromatin was solubilized by sonication using a Bioruptor. b-dUTPlabeled chromatin was purified on streptavidin-coated magnetic beads. Total chromatin (input) and isolated nascent chromatin was decrosslinked by boiling for $40 \mathrm{~min}$ in LSB $(50 \mathrm{mM}$ Tris- $\mathrm{HCl}$ at $\mathrm{pH} 6.8,100 \mathrm{mM}$ DTT, 2\% SDS, 8\% glycerol, Bromphenol blue).

\section{Mass spectrometry analysis of solubilized chromatin}

Histone bands were separated by SDS-PAGE, stained with Coomassie (Brilliant blue G-250), and excised at appropriate heights. Bands were destained in $50 \%$ acetonitrile $/ 50 \% 100 \mathrm{mM}$ ammonium bicarbonate. Histones were chemically modified by propionylation $(30 \mathrm{~min}$ at room temperature, $2.5 \%$ propionic anhydride [Sigma] in ammonium bicarbonate at $\mathrm{pH} 7.5)$ to prevent tryptic cleavage. Histone proteins were then digested with $200 \mathrm{ng}$ of trypsin (Promega) in $50 \mathrm{mM}$ ammonium bicarbonate overnight, and the supernatant was desalted by carbon TopTips (Glygen) according to the manufacturer's instructions. The peptides were injected into an Ultimate 3000 high-performance liquid chromatography (HPLC) system (LC Packings Dionex) and separated with a gradient from $5 \%$ to $60 \%$ acetonitrile in $0.1 \%$ formic acid over $40 \mathrm{~min}$ at $300 \mathrm{~nL} /$ min on a $75-\mu \mathrm{m} \mathrm{ID} \times 10-\mathrm{cm}$ ReproSil-Pur C1-AQ analytical column (2.4-

$\mu \mathrm{m})$ (Dr. Maisch GmbH). The effluent from the HPLC was directly electrosprayed into the LTQ Orbitrap XL mass spectrometer (Thermo Fisher Scientific). The mass spectrometry instrument was operated in the data-dependent mode to automatically switch between full-scan mass spectrometry and tandem mass spectrometry acquisition. Survey full-scan spectra $(\mathrm{m} / \mathrm{z}$ 250-2000) were acquired in the Orbitrap with resolution $R=60,000$ at $m / z 400$. For all measurements with the Orbitrap detector, three lock mass ions from ambient air $(\mathrm{m} / \mathrm{z}=$ $371.10123,445.12002$, and 519.13882) were used for internal calibration as described (Olsen et al. 2005). The six most intense peptide ions with charge states between 2 and 5 were sequentially isolated (window $=2.0 \mathrm{~m} / \mathrm{z}$ ) to a target value of 10,000 and fragmented in the linear ion trap by collision-induced dissociation (CID). Fragment ion spectra were recorded in the linear trap of the instrument. A dynamic exclusion time of $180 \mathrm{sec}$ was applied. Typical mass spectrometric conditions were spray voltage $1.4 \mathrm{kV}$, no sheath and auxiliary gas flow, heated capillary temperature $200^{\circ} \mathrm{C}$, and normalized collision energy $35 \%$ for CID in the linear ion trap. An activation $Q=0.25$ and activation time of 30 msec were used. Data analysis was performed with XCalibur Qual browser software (Thermo Fisher Scientific) by using doubly and triply charged peptide masses for extracted ion chromatograms (XICs). XICs were checked manually, and values were exported to Excel for further calculations. The mass spectrometry raw data were deposited to the ProteomeXchange Consortium with the data set identifier px-submission number 37929.

\section{Histone variants}

Unique heavy and light peptides were used to identify histone variants. $\mathrm{H} 3.1$ and $\mathrm{H} 3.3$ differ in amino acids $31,87,89$, and 90 . The tryptic peptide containing amino acids 87,89 , and 90 (amino acids $84-116$ ) is difficult to detect; therefore, the unmodified peptide comprising amino acids $27-40$ was taken for quantitation of the H3.3 variant. H2A was identified by the peptide KGNYAER (amino acids 38-44), H2A.X was identified by the peptide KGHYAER (38-44), and H2A.Z was identified by the peptide HLKSR (37-41). The proportion of each canonical and variant histone is expressed as a percentage of all detected variants (H2A: H2A+H2A.X $+\mathrm{H} 2 \mathrm{~A} . \mathrm{Z}$; and H3: H3.1+H3.3 or the common peptide H3 41-49).

\section{Statistical analysis}

All diagrams show the mean of biological independent experiments, with error bars indicating standard deviation. For statistical analysis, unpaired $t$-test was performed using Prism.6. $P$-values are indicated by asterisks $\left(P<0.0001\left[{ }^{* * * *}\right], P<0.001\left[{ }^{* *}\right], P<0.01\left[{ }^{* *}\right]\right.$, and $\left.P<0.05\left[{ }^{*}\right]\right)$, and n.s. indicates nonsignificant.

\section{Acknowledgments}

We thank Carl Wu, Konrad Hochedlinger, Kristian Helin, and Peter Becker for critical reading of this manuscript, and Zuzana Jasencakova and Cristina Gonzalez Aguilera for technical support. This work was supported by a European Research Council Starting Grant (ERC2011StG, no. 281,765 to A.G.), the Danish National Research Foundation to the Center for Epigenetics (DNRF82 to S.S., O.N.J., and A.G.), the Danish Cancer Society (A.G.), and the Lundbeck Foundation (A.G.). A.G. is an EMBO Young Investigator. The work in A.I.'s laboratory is supported by grants from the European Union (EpiGeneSys 2570829) and the German Research Council (DFG SFB 1064-Z3). C.A. was supported by fellowships from Human Frontier Science Program and the Danish Medical Research Council.

\section{References}

Aagaard L, Laible G, Selenko P, Schmid M, Dorn R, Schotta G, Kuhfittig S, Wolf A, Lebersorger A, Singh PB, et al. 1999. Functional mammalian homologues of the Drosophila PEV-modifier Su(var)3-9 encode centromere-associated proteins which complex with the heterochromatin component M31. EMBO J 18: 1923-1938. 
Alabert C, Groth A. 2012. Chromatin replication and epigenome maintenance. Nat Rev Mol Cell Biol 13: 153-167.

Alabert C, Bukowski-Wills JC, Lee SB, Kustatscher G, Nakamura K, de Lima Alves F, Menard P, Mejlvang J, Rappsilber J, Groth A. 2014. Nascent chromatin capture proteomics determines chromatin dynamics during DNA replication and identifies unknown fork components. Nat Cell Biol 16: 281-293.

Annunziato AT. 2005. Split decision: what happens to nucleosomes during DNA replication? J Biol Chem 280: 12065-12068.

Apostolou E, Hochedlinger K. 2013. Chromatin dynamics during cellular reprogramming. Nature 502: 462-471.

Benson LJ, Gu Y, Yakovleva T, Tong K, Barrows C, Strack CL, Cook RG, Mizzen CA, Annunziato AT. 2006. Modifications of H3 and H4 during chromatin replication, nucleosome assembly, and histone exchange. I Biol Chem 281: 9287-9296.

Campos EI, Stafford JM, Reinberg D. 2014. Epigenetic inheritance: histone bookmarks across generations. Trends Cell Biol.

Deal RB, Henikoff JG, Henikoff S. 2010. Genome-wide kinetics of nucleosome turnover determined by metabolic labeling of histones. Science 328: 1161-1164.

Di Micco R, Sulli G, Dobreva M, Liontos M, Botrugno OA, Gargiulo G, dal Zuffo R, Matti V, d'Ario G, Montani E, et al. 2011. Interplay between oncogene-induced DNA damage response and heterochromatin in senescence and cancer. Nat Cell Biol 13: 292-302.

Fisher D, Mechali M. 2003. Vertebrate HoxB gene expression requires DNA replication. $Е M B O$ I 22: 3737-3748.

Gaydos LJ, Wang W, Strome S. 2014. Gene repression. H3K27me and PRC2 transmit a memory of repression across generations and during development. Science 345: 1515-1518.

Guo S, Zi X, Schulz VP, Cheng J, Zhong M, Koochaki SH, Megyola CM, Pan X, Heydari K, Weissman SM, et al. 2014. Nonstochastic reprogramming from a privileged somatic cell state. Cell 156: 649-662.

Hansen KH, Bracken AP, Pasini D, Dietrich N, Gehani SS, Monrad A, Rappsilber J, Lerdrup M, Helin K. 2008. A model for transmission of the H3K27me3 epigenetic mark. Nat Cell Biol 10: 1291-1300.

Huang C, Xu M, Zhu B. 2013. Epigenetic inheritance mediated by histone lysine methylation: maintaining transcriptional states without the precise restoration of marks? Philos Trans $R$ Soc Lond B Biol Sci 368: 20110332.

Jasencakova Z, Scharf AN, Ask K, Corpet A, Imhof A, Almouzni G, Groth A. 2010. Replication stress interferes with histone recycling and predeposition marking of new histones. Mol Cell 37: 736-743.

Kalb R, Latwiel S, Baymaz HI, Jansen PW, Muller CW, Vermeulen M, Muller J. 2014. Histone H2A monoubiquitination promotes histone H3 methylation in Polycomb repression. Nat Struct Mol Biol 21: 569-571.

Kouzarides T. 2007. Chromatin modifications and their function. Cell 128: 693-705.

Lande-Diner L, Zhang J, Cedar H. 2009. Shifts in replication timing actively affect histone acetylation during nucleosome reassembly. Mol Cell 34: $767-774$

Loyola A, Bonaldi T, Roche D, Imhof A, Almouzni G. 2006. PTMs on H3 variants before chromatin assembly potentiate their final epigenetic state. Mol Cell 24: 309-316.

Luk E, Ranjan A, Fitzgerald PC, Mizuguchi G, Huang Y, Wei D, Wu C. 2010. Stepwise histone replacement by SWR1 requires dual activation with histone H2A.Z and canonical nucleosome. Cell 143: 725-736.

Margueron R, Reinberg D. 2010. Chromatin structure and the inheritance of epigenetic information. Nat Rev Genet 11: 285-296.

Margueron R, Justin N, Ohno K, Sharpe ML, Son J, Drury WJIII, Voigt P, Martin SR, Taylor WR, De Marco V, et al. 2009. Role of the polycomb protein EED in the propagation of repressive histone marks. Nature 461: 762-767.

McCabe MT, Graves AP, Ganji G, Diaz E, Halsey WS, Jiang Y, Smitheman KN, Ott HM, Pappalardi MB, Allen KE, et al. 2012. Mutation of A677 in histone methyltransferase EZH2 in human B-cell lymphoma promotes hypertrimethylation of histone H3 on lysine 27 (H3K27). Proc Natl Acad Sci 109: 2989-2994.

Nekrasov M, Amrichova J, Parker BJ, Soboleva TA, Jack C, Williams R, Huttley GA, Tremethick DJ. 2012. Histone H2A.Z inheritance during the cell cycle and its impact on promoter organization and dynamics. Nat Struct Mol Biol 19: 1076-1083.

Olsen JV, de Godoy LM, Li G, Macek B, Mortensen P, Pesch R, Makarov A, Lange O, Horning S, Mann M. 2005. Parts per million mass accuracy on an Orbitrap mass spectrometer via lock mass injection into a C-trap. Mol Cell Proteomics 4: 2010-2021.

Pengelly AR, Copur O, Jackle H, Herzig A, Muller J. 2013. A histone mutant reproduces the phenotype caused by loss of histone-modifying factor Polycomb. Science 339: 698-699.

Pesavento JJ, Yang H, Kelleher NL, Mizzen CA. 2008. Certain and progressive methylation of histone $\mathrm{H} 4$ at lysine 20 during the cell cycle. Mol Cell Biol 28: 468-486.

Petruk S, Sedkov Y, Johnston DM, Hodgson JW, Black KL, Kovermann SK, Beck S, Canaani E, Brock HW, Mazo A. 2012. TrxG and PcG proteins but not methylated histones remain associated with DNA through replication. Cell 150: 922-933.

Petruk S, Black KL, Kovermann SK, Brock HW, Mazo A. 2013. Stepwise histone modifications are mediated by multiple enzymes that rapidly associate with nascent DNA during replication. Nat Commun 4: 2841.

Probst AV, Dunleavy E, Almouzni G. 2009. Epigenetic inheritance during the cell cycle. Nat Rev Mol Cell Biol 10: 192-206.

Scharf AN, Barth TK, Imhof A. 2009. Establishment of histone modifications after chromatin assembly. Nucleic Acids Res 37: 50325040.

Silva MC, Bodor DL, Stellfox ME, Martins NM, Hochegger H, Foltz DR, Jansen LE. 2012. Cdk activity couples epigenetic centromere inheritance to cell cycle progression. Dev Cell 22: 52-63.

Skene PJ, Henikoff S. 2013. Histone variants in pluripotency and disease. Development 140: 2513-2524.

Sobel RE, Cook RG, Perry CA, Annunziato AT, Allis CD. 1995. Conservation of deposition-related acetylation sites in newly synthesized histones H3 and H4. Proc Natl Acad Sci 92: 1237-1241.

Steffen PA, Ringrose L. 2014. What are memories made of? How Polycomb and Trithorax proteins mediate epigenetic memory. Nat Rev Mol Cell Biol 15: 340-356.

Sweet SM, Li M, Thomas PM, Durbin KR, Kelleher NL. 2010. Kinetics of re-establishing H3K79 methylation marks in global human chromatin. J Biol Chem 285: 32778-32786.

Taddei A, Roche D, Sibarita JB, Turner BM, Almouzni G. 1999. Duplication and maintenance of heterochromatin domains. J Cell Biol 147: $1153-1166$.

Tsubouchi T, Soza-Ried J, Brown K, Piccolo FM, Cantone I, Landeira D, Bagci H, Hochegger H, Merkenschlager M, Fisher AG. 2013. DNA synthesis is required for reprogramming mediated by stem cell fusion. Cell 152: $873-883$

Xu M, Wang W, Chen S, Zhu B. 2012. A model for mitotic inheritance of histone lysine methylation. EMBO Rep 13: 60-67.

Zee BM, Britton LM, Wolle D, Haberman DM, Garcia BA. 2012. Origins and formation of histone methylation across the human cell cycle. Mol Cell Biol 32: 2503-2514.

Zhang J, Xu F, Hashimshony T, Keshet I, Cedar H. 2002. Establishment of transcriptional competence in early and late S phase. Nature 420: 198-202. 


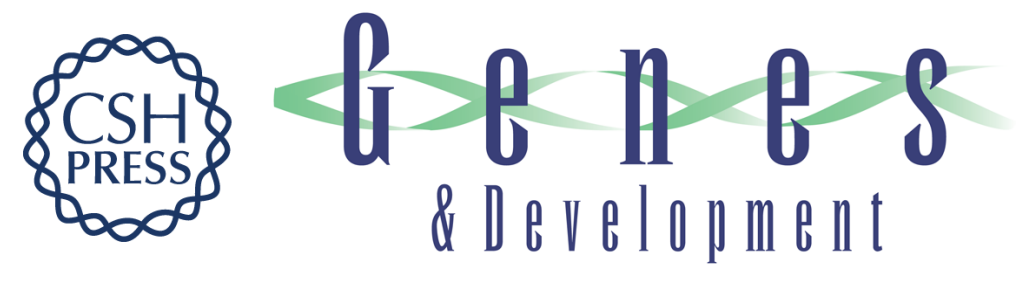

\section{Two distinct modes for propagation of histone PTMs across the cell cycle}

Constance Alabert, Teresa K. Barth, Nazaret Reverón-Gómez, et al.

Genes Dev. 2015, 29:

Access the most recent version at doi:10.1101/gad.256354.114

\section{Supplemental http://genesdev.cshlp.org/content/suppl/2015/03/18/29.6.585.DC1 Material}

References This article cites 40 articles, 16 of which can be accessed free at: http://genesdev.cshlp.org/content/29/6/585.full.html\#ref-list-1

Creative This article is distributed exclusively by Cold Spring Harbor Laboratory Press for the first Commons six months after the full-issue publication date (see

License http://genesdev.cshlp.org/site/misc/terms.xhtml). After six months, it is available under a Creative Commons License (Attribution-NonCommercial 4.0 International), as described at http://creativecommons.org/licenses/by-nc/4.0/.

Email Alerting Receive free email alerts when new articles cite this article - sign up in the box at the top Service right corner of the article or click here.

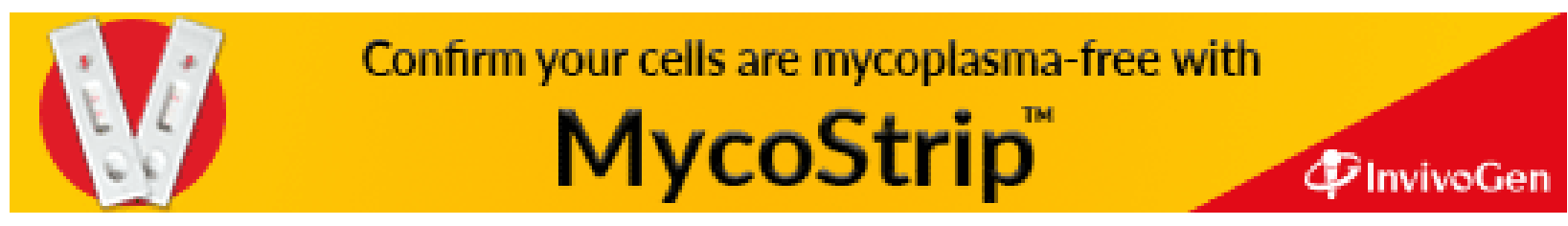

